\title{
Exact Capacity Distribution for Dual MIMO Systems in Ricean Fading
}

\author{
Peter J. Smith, Member, IEEE, and Lee M. Garth, Member, IEEE
}

\begin{abstract}
It is well known that multiple input multiple output (MIMO) systems offer the promise of achieving very high spectrum efficiencies (many tens of $\mathrm{bit} / \mathrm{s} / \mathrm{Hz}$ ) in a mobile environment. The gains in MIMO capacity are sensitive to the type of channel encountered in the radio environment. To date most analytical work has concentrated on Rayleigh fading channels. Hence, in this letter we consider the capacity outage performance of MIMO systems in Ricean channels. Due to analytical complexity we concentrate on dual antenna systems (either two transmit or two receive antennas) and derive exact densities and distribution functions for the capacity.
\end{abstract}

Index Terms-Information rates, multiple input multiple output (MIMO) systems, wireless channel models, .

\section{INTRODUCTION}

$\mathbf{S}_{\mathrm{T}}$ INCE the pioneering work of Foschini and Gans [1] and Telatar [2], multiple input multiple output (MIMO) systems have received considerable attention in recent years as they have the potential to provide quantum leaps in capacity. The capacity of MIMO systems has been intensively studied, but analytical results are mainly available for Rayleigh fading or asymptotic scenarios. For the non-Rayleigh case, simulations are usually required. The Ricean case has been discussed in [3], [4], but few analytical results are available. In particular, we have not found any exact results for capacity distributions in the literature. Hence, in Section III we derive the exact density and distribution function for the MIMO capacity in a Ricean channel where the number of transmit or receive antennas is limited to two. Firstly, in Section II we describe the system model and some link capacity results. After the derivations we give results and conclusions in Section IV.

\section{SySTEM MOdEL AND LinK CAPACITY}

In this letter we assume a single-user MIMO system with no channel state information (CSI) at the transmitter, perfect CSI at the receiver, and employing equal power transmission over a flat Ricean fading channel. For such a system with $n_{T}$ transmit and $n_{R}$ receive antennas the received signal is:

$$
\boldsymbol{r}=\boldsymbol{H} \boldsymbol{s}+\boldsymbol{n}
$$

where $\boldsymbol{r}$ is the $n_{R} \times 1$ received signal vector, $\boldsymbol{s}$ is the complex $n_{T} \times 1$ transmitted signal vector and $\boldsymbol{H}$ is an $n_{R} \times n_{T}$ complex channel gain matrix. The additive white Gaussian noise

Manuscript received April 17, 2003. The associate editor coordinating the review of this letter and approving it for publication was Dr. S. Diggavi.

The authors are with the Department of Electrical and Computer Engineering, University of Canterbury, Christchurch, New Zealand (e-mail: p.smith@elec.canterbury.ac.nz; 1.garth@elec.canterbury.ac.nz).

Digital Object Identifier 10.1109/LCOMM.2003.822515
(AWGN) vector $n$ consists of $n_{R}$ independent noise components of modulus variance normalized to 1 .

In Ricean fading the elements of $\boldsymbol{H}$ are nonzero mean, complex Gaussians. Hence, we can express $\boldsymbol{H}$ in matrix notation [4] as

$$
\boldsymbol{H}=a \boldsymbol{H}^{\mathrm{sp}}+b \boldsymbol{H}^{\mathrm{sc}}
$$

where the specular and scattered components of $H$ are denoted by superscripts. Matrix $\boldsymbol{H}^{\mathrm{sp}}$ is deterministic with unit magnitude elements, and the entries of $\boldsymbol{H}^{\mathrm{sc}}$ are independent, zero mean, unit variance, complex Gaussians. The parameters $a$ and $b$ satisfy $a_{2}+b_{2}=1$ so that the SNR is not scaled by the channel. In standard models [4] the specular matrix is defined as:

$$
\boldsymbol{H}^{\mathrm{sp}}=\boldsymbol{a}\left(\theta_{r}\right) \boldsymbol{a}\left(\theta_{t}\right)^{T}
$$

where $\boldsymbol{a}\left(\theta_{r}\right)$ and $\boldsymbol{a}\left(\theta_{t}\right)$ are the specular array responses at the receiver and transmitter. If a $k$-element array is linear, the response is $\boldsymbol{a}(\theta)=\left[1 e^{j 2 \pi d \cos (\theta)} \cdots e^{j 2 \pi d(k-1) \cos (\theta)}\right]^{T}$, where $\theta$ is the angle of arrival or departure of the specular component, and $d$ is the antenna spacing in wavelengths. This form gives the specular matrix a rank of one. We will use this model in our numerical results, although our analysis holds for more general forms of $\boldsymbol{H}^{\mathrm{sp}}$. The strength of the LOS component is measured using the $K$-factor

$$
K=10 \log _{10}\left(\frac{a^{2}}{b^{2}}\right) \mathrm{dB}
$$

The capacity of such a system is now very well known [1], [2] and is given by

$$
C=\log _{2}\left[\operatorname{det}\left(\boldsymbol{I}_{m}+\frac{\rho}{n_{T}} \widetilde{\boldsymbol{W}}\right)\right]
$$

where $\boldsymbol{I}_{m}$ is the $m \times m$ identity matrix, $\rho$ is the average signal-to-noise ratio (SNR) per receive antenna, $m=\min \left(n_{R}, n_{T}\right), n=\max \left(n_{R}, n_{T}\right)$, and the $m \times m$ matrix $\widetilde{W}$ is given by

$$
\widetilde{\boldsymbol{W}}= \begin{cases}\boldsymbol{H} \boldsymbol{H}^{\dagger}, & \text { for } n_{R} \leq n_{T} \\ \boldsymbol{H}^{\dagger} \boldsymbol{H}, & \text { for } n_{T}<n_{R}\end{cases}
$$

With these definitions we can rewrite the channel capacity as

$$
C=\log _{2}\left[\prod_{i=1}^{m}\left(1+\frac{\rho b^{2}}{n_{T}} w_{i}\right)\right] .
$$

where $\left(w_{1}, w_{2}, \ldots, w_{m}\right)$ are the eigenvalues of $\boldsymbol{W}=\widetilde{\boldsymbol{W}} / b^{2}$. We also need to define the associated LOS version of (6) where the matrix $\boldsymbol{M}$ is given by:

$$
\boldsymbol{M}= \begin{cases}\frac{a^{2}}{b^{2}} \boldsymbol{H}^{\mathrm{sp}} \boldsymbol{H}^{s p \dagger}, & \text { for } n_{R} \leq n_{T} \\ \frac{a^{2}}{b^{2}} \boldsymbol{H}^{s p \dagger} \boldsymbol{H}^{\mathrm{sp}}, & \text { for } n_{T}<n_{R} .\end{cases}
$$


The eigenvalues of $\boldsymbol{M}$ are denoted by $\left(f_{1}, f_{2}, \ldots, f_{m}\right)$. In the rank one standard model for $\boldsymbol{M}$, these eigenvalues are given by: $f_{1}=\left(a^{2} / b^{2}\right) m n$ and $f_{2}=f_{3}=\ldots=f_{m}=0$.

\section{DERIVATIONS}

To derive the density of $C$, we need the following results. The joint density of the ordered eigenvalues of $\boldsymbol{W}, w_{1}<w_{2}<$ $\ldots<w_{m}$, is given by James [5] as:

$$
\begin{aligned}
f(\boldsymbol{w})= & \exp \left[-\sum_{i=1}^{m}\left(f_{i}+w_{i}\right)\right]{ }_{0} \widetilde{F}_{1}(n ; f, \boldsymbol{w}) \prod_{i=1}^{m}\left(w_{i}^{n-m}\right) \\
& \times \prod_{i<j}\left(w_{i}-w_{j}\right)^{2}\left[\prod_{i=1}^{m}(n-i) !(m-i) !\right]^{-1}
\end{aligned}
$$

where $\boldsymbol{w}=\left(w_{1}, w_{2}, \ldots, w_{m}\right)$ and $\boldsymbol{f}=\left(f_{1}, f_{2}, \ldots, f_{m}\right)$. The function ${ }_{0} \widetilde{F}_{1}(\cdot)$ is a hypergeometric function of two matrix arguments. For the case of unequal $f_{i}$ 's, a convenient version of this function is given by Gross and Richards [6] as:

$$
{ }_{0} \widetilde{F}_{1}(n ; \boldsymbol{f}, \boldsymbol{w})=\prod_{i=1}^{m}\left[\frac{(n-i) !}{(n-m) !}\right] \frac{\left.\right|_{0} \mathcal{F}_{1}\left(n-m+1, f_{i} w_{j}\right) \mid}{\alpha(f) \prod_{i<j}\left(w_{i}-w_{j}\right)}
$$

where $|A(i, j)|$ indicates the determinant of the $m \times m$ matrix with $(i, j)$-th element given by $A(i, j)$ and in the denominator, $\alpha(f)=\Pi_{i<j}\left(f_{i}-f_{j}\right) / \Pi_{i=1}^{m}(i-1)$ !. The function ${ }_{0} \mathcal{F}_{1}(n-$ $m+1, x)$ in (10) is defined in [7] as

$$
{ }_{0} \mathcal{F}_{1}(n-m+1, x)=(n-m) ! x^{-\frac{(n-m)}{2}} I_{n-m}(2 \sqrt{x})
$$

where $I_{n-m}(\cdot)$ is a modified Bessel function.

\section{A. SIMO or MISO Systems}

For completeness we give results for $m=1$, corresponding either to a single input multiple output (SIMO) system or a multiple input single output (MISO) system. Here, the channel gain matrix collapses to either a row or column vector $h$, and the capacity (5) reduces to

$$
C=\log _{2}\left[1+\frac{\rho}{n_{t}} \sum_{i=1}^{n}\left|h_{i}\right|^{2}\right] .
$$

Each term $\left(1 / b^{2}\right)=\left|h_{i}\right|^{2}$ is an independent complex noncentral chi-squared random variable with one degree of freedom and noncentrality parameter $a^{2} / b^{2}$ for specular matrix (3). Thus, the sum $Y=\left(1 / b^{2}\right) \sum_{i=1}^{n}\left|h_{i}\right|^{2}$ is a complex noncentral chisquared random variable with $n$ degrees of freedom and noncentrality parameter $f_{1}=\left(a^{2} / b^{2}\right) n$, the scalar version of $\boldsymbol{M}$ corresponding to the $m=1$ case. The density and distribution of $Y$ are [8]

$$
\begin{aligned}
& f_{Y}(y)=\left(\frac{y}{f_{1}}\right)^{\frac{(n-1)}{2}} e^{-\left(y+f_{1}\right)} I_{n-1}\left(2 \sqrt{f_{1} y}\right) \\
& F_{Y}(y)=1-Q_{n}\left(\sqrt{2 f_{1}}, \sqrt{2 y}\right)
\end{aligned}
$$

where $y>0$ and $Q_{n}(\cdot)$ is the generalized Marcum $Q$-function. Hence, for $c>0$ the density and distribution of $C$ are

$$
\begin{aligned}
& f_{C}(c)=\ln 2\left(\frac{n_{T}}{\rho b^{2}}\right) 2^{c} f_{Y}\left(\frac{n_{T}}{\rho b^{2}}\left[2^{c}-1\right]\right) \\
& F_{C}(c)=F_{Y}\left(\frac{n_{T}}{\rho b^{2}}\left[2^{c}-1\right]\right) .
\end{aligned}
$$

\section{B. Dual Input or Dual Output Systems}

Here we have $m=2$, corresponding to either $n_{T}=2$ or $n_{R}=2$. We assume $n \geq 2$ since the single antenna case was given above. Rewriting (9) for the $m=2$ case using (10) and (11), we have

$$
\begin{aligned}
f\left(w_{1}, w_{2}\right)= & \frac{e^{-\left(f_{1}+f_{2}\right)}\left(w_{1} w_{2}\right)^{n-2}\left(w_{1}-w_{2}\right) e^{-\left(w_{1}+w_{2}\right)}}{2\left(f_{1}-f_{2}\right)\left(f_{1} w_{1} f_{2} w_{2}\right)^{\frac{(n-2)}{2}}} \\
& \times\left[I_{n-2}\left(2 \sqrt{f_{1} w_{1}}\right) I_{n-2}\left(2 \sqrt{f_{2} w_{2}}\right)\right. \\
& \left.-I_{n-2}\left(2 \sqrt{f_{1} w_{2}}\right) I_{n-2}\left(2 \sqrt{f_{2} w_{1}}\right)\right]
\end{aligned}
$$

with a factor of $1 / 2$ incorporated to change to unordered eigenvalues $0 \leq w_{1}, w_{2}$. But, as mentioned after (8), we are particularly interested in a rank 1 structure for $\boldsymbol{M}$, yielding $f_{1}>0$ and $f_{2}=0$. Setting $f_{2}=0$, we use the relation

$$
\lim _{f \rightarrow 0} \frac{I_{n-2}(2 \sqrt{f w})}{f^{\frac{(n-2)}{2}}}=\frac{w^{\frac{(n-2)}{2}}}{(n-2) !}
$$

to reduce (15) to

$$
\begin{aligned}
f\left(w_{1}, w_{2}\right)= & \frac{e^{-f_{1}}}{2(n-2) ! f_{1}^{\frac{n}{2}}}\left[\left(\sqrt{w_{1}} w_{2}\right)^{n-2}\left(w_{1}-w_{2}\right) e^{-\left(w_{1}+w_{2}\right)}\right. \\
& \times I_{n-2}\left(2 \sqrt{f_{1} w_{1}}\right)-\left(w_{1} \sqrt{w_{2}}\right)^{n-2} \\
& \left.\times\left(w_{1}-w_{2}\right) e^{-\left(w_{1}+w_{2}\right)} I_{n-2}\left(2 \sqrt{f_{1} w_{2}}\right)\right] .
\end{aligned}
$$

Applying the required coordinate transformations, the density of

$$
C=\log _{2}\left[\left(1+\frac{w_{1}}{\gamma}\right)\left(1+\frac{w_{2}}{\gamma}\right)\right]
$$

for $c>0$ and $\gamma=n_{T} /\left(\rho b^{2}\right)$ is given by

$$
\begin{aligned}
f_{C}(c) & \\
= & \frac{2^{c} \ln 2 \gamma^{3} e^{-f_{1}}}{2(n-2) ! f_{1}^{\frac{n}{2}}} \\
& \times \int_{1}^{2^{c}}\left\{\left[\sqrt{\gamma\left(\frac{2^{c}}{v}-1\right)} \gamma(v-1)\right]^{n-2}\left(\frac{2^{c}}{v^{2}}-1\right)\right. \\
& \times e^{-\gamma\left[\frac{2^{c}}{v}+v-2\right]} I_{n-2}\left(2 \sqrt{f_{1} \gamma\left(\frac{2^{c}}{v}-1\right)}\right) \\
& -\left[\gamma\left(\frac{2^{c}}{v}-1\right) \sqrt{\gamma(v-1)}\right]^{n-2}\left(\frac{2^{c}}{v^{2}}-1\right) \\
& \left.\times e^{-\gamma\left[\frac{2^{c}}{v}+v-2\right]} I_{n-2}\left(2 \sqrt{f_{1} \gamma(v-1)}\right)\right\} d v .
\end{aligned}
$$

To the best of our knowledge, the evaluation of (17) requires numerical integration. Integrating the density, we obtain the distribution function of $C$ as shown in (18) at the bottom of the next page. This is a double integral, but fortuitously one of the integrals can be evaluated. Setting $x=\gamma((u / v)-1)$ in the first integral over $u$ in (18), we can write this integral as (19), shown at the bottom of the next page. To produce the final form of $\psi_{1}(v)$, we used the recursion

$$
I_{m-1}(z)=\frac{2 m}{z} I_{m}(z)+I_{m+1}(z) .
$$


Likewise, setting $x=\gamma((u / v)-1)$ in the second integral over $u$ in (18), we can write this integral as

$$
\begin{aligned}
\psi_{2}(v) \triangleq & \frac{v}{\gamma} \int_{0}^{\gamma\left(\frac{2^{c}}{v}-1\right)} x^{n-2}[x-\gamma(v-1)] e^{-x} d x \\
= & \frac{v}{\gamma} e^{-\gamma\left(\frac{2^{c}}{v}-1\right)} \\
& \times\left\{\gamma(v-1) \sum_{r=0}^{n-2} \frac{(n-2) !}{(n-2-r) !}\left[\gamma\left(\frac{2^{c}}{v}-1\right)\right]^{n-2-r}\right. \\
& \left.-\sum_{r=0}^{n-1} \frac{(n-1) !}{(n-1-r) !}\left[\gamma\left(\frac{2^{c}}{v}-1\right)\right]^{n-1-r}\right\} \\
& +\frac{v}{\gamma}[(n-1) !-\gamma(v-1)(n-2) !] .
\end{aligned}
$$

Using (19) and (20) in (18), we finally write the distribution of $C$ as

$$
\begin{aligned}
F_{C}(c)= & \frac{\gamma^{2}}{2(n-2) ! f_{1}} \int_{1}^{2^{c}}\left\{\frac{\psi_{1}(v)}{v}[\gamma(v-1)]^{n-2}\right. \\
& \times e^{-\gamma(v-1)}-\frac{\psi_{2}(v)}{v}\left[\frac{\gamma(v-1)}{f_{1}}\right]^{\frac{n-2}{2}} \\
& \left.\times e^{-\left[\gamma(v-1)+f_{1}\right]} I_{n-2}\left(2 \sqrt{f_{1} \gamma(v-1)}\right)\right\} d v .
\end{aligned}
$$

Hence, the final form of the distribution function requires a single numerical integration and allows the direct computation of capacity outage probabilities.

Finally, note that density (17) and distribution (21) were derived for the rank one standard model for $\boldsymbol{M}$ with $f_{2}=0$. Applying the required coordinate transformations directly to (15) it is trivial to find the density of $C$ for $f_{1}>f_{2}>0$. To obtain analogous simplified expressions for the distribution function requires further investigation.

\section{Simulation Results ANd CONCLUSIONS}

To verify our analysis, we have calculated the capacity distribution for $(1,2),(1,4),(2,2)$ and $(2,4)$ MIMO systems. Fig. 1 shows the capacity distributions, calculated from (14) and (21) with $\theta_{t}=20^{\circ}, \theta_{r}=35^{\circ}, d=4, \rho=10 \mathrm{~dB}$, and $K=$ $10 \mathrm{~dB}$, along with corresponding Monte Carlo simulation re-

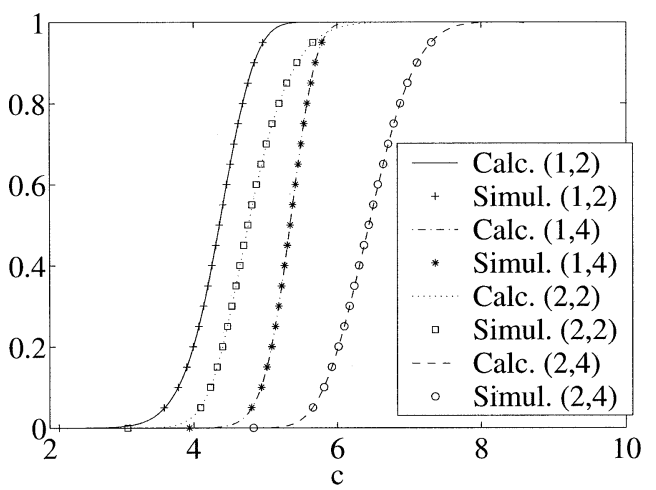

Fig. 1. Capacity distributions for $(1,2),(1,4),(2,2),(2,4)$ MIMO systems from analysis and simulation.

sults. Here the trial parameters were selected to verify our analysis rather than to model a particular array environment. An antenna spacing of $d=4$ wavelengths would correspond to a base station. The simulated distributions are based on 20000 Monte Carlo samples. Our theoretical distributions line up exactly with the simulation results. Hence, we have derived the exact density and distribution function for MIMO capacity in a flat Ricean fading channel where $m \leq 2$.

\section{REFERENCES}

[1] G. J. Foschini and M. J. Gans, "On limits of wireless communication in a fading environment when using multiple antennas," Wireless Personal Commun., vol. 6, no. 3, pp. 311-335, Mar. 1998.

[2] I. E. Telatar, "Capacity of multi-antenna Gaussian channels," European Trans. on Telecomm. Related Technol., vol. 10, pp. 585-595, Nov.-Dec. 1999.

[3] P. Driessen and G. J. Foschini, "On the capacity formula for multipleinput-multiple-output wireless channels: a geometric approach," IEEE Trans. Commun., vol. 47, no. 2, pp. 173-176, Mar. 1999.

[4] F. R. Farrokhi, G. J. Foschini, A. Lozano, and R. Valenzuela, "Linkoptimal space-time processing with multiple transmit and receive antennas," IEEE Commun. Lett., vol. 5, no. 3, pp. 85-87, Mar. 2001.

[5] A. T. James, "Distributions of matrix variates and latent roots derived from normal samples," Ann. Math. Statist., vol. 35, no. 1, pp. 475-501, 1964.

[6] K. I. Gross and D. S. P. Richards, "Total positivity, spherical series, and hypergeometric functions of matrix argument," Journal of Approximation Theory, vol. 59, pp. 224-246, 1989.

[7] J. Spanier and K. B. Oldham, Eds., An Atlas of Functions. New York: Hemisphere Publishing Corporation, 1987.

[8] R. A. Fisher, "The general sampling distribution of the multiple correlation coefficient," in Proc. Roy. Soc. London Ser. A, vol. 121, 1928, pp. 654-673.

$$
\begin{aligned}
F_{C}(c)= & \frac{\gamma^{2}}{2(n-2) ! f_{1}}\left\{\int _ { 1 } ^ { 2 ^ { c } } \frac { 1 } { v } [ \gamma ( v - 1 ) ] ^ { n - 2 } e ^ { - \gamma ( v - 1 ) } \int _ { v } ^ { 2 ^ { c } } [ \frac { \gamma ( \frac { u } { v } - 1 ) } { f _ { 1 } } ] ^ { \frac { n - 2 } { 2 } } \gamma ( \frac { u } { v } - v ) e ^ { - [ \gamma ( \frac { u } { v } - 1 ) + f _ { 1 } ] } I _ { n - 2 } \left(2 \sqrt{\left.f_{1} \gamma\left(\frac{u}{v}-1\right)\right)} d u d v\right.\right. \\
& \left.\quad \times \int_{1}^{2^{c}} \frac{1}{v}\left[\frac{\gamma(v-1)}{f_{1}}\right]^{\frac{n-2}{2}} e^{-\left[\gamma(v-1)+f_{1}\right]} I_{n-2}\left(2 \sqrt{f_{1} \gamma(v-1)}\right) \int_{v}^{2^{c}}\left[\gamma\left(\frac{u}{v}-1\right)\right]^{n-2} \gamma\left(\frac{u}{v}-v\right) e^{-\gamma\left(\frac{u}{v}-1\right)} d u d v\right\} \\
\psi_{1}(v) \triangleq & \frac{v}{\gamma} \int_{0}^{\gamma\left(\frac{2^{c}}{v}-1\right)}\left[\frac{x}{f_{1}}\right]^{\frac{n-2}{2}}[x-\gamma(v-1)] e^{-\left(x+f_{1}\right)} I_{n-2}\left(2 \sqrt{f_{1} x}\right) d x \\
= & \frac{(n-1) v}{\gamma}\left[Q_{n}\left(\sqrt{2 f_{1}}, 0\right)-Q_{n}\left(\sqrt{2 f_{1}}, \sqrt{2 \gamma\left(\frac{2^{c}}{v}-1\right)}\right)\right]+\frac{v f_{1}}{\gamma}\left[Q_{n+1}\left(\sqrt{2 f_{1}}, 0\right)-Q_{n+1}\left(\sqrt{2 f_{1}}, \sqrt{2 \gamma\left(\frac{2^{c}}{v}-1\right)}\right)\right] \\
& -v(v-1)\left[Q_{n-1}\left(\sqrt{2 f_{1}}, 0\right)-Q_{n-1}\left(\sqrt{2 f_{1}}, \sqrt{2 \gamma\left(\frac{2^{c}}{v}-1\right)}\right)\right]
\end{aligned}
$$

